\title{
\begin{tabular}{l|l} 
POLITIQUES \& & Politiques et management public
\end{tabular}

\section{La régionalisation ferroviaire en Suisse : la performance sans la compétition. Un exemple pour la France?}

Regionalization railway in Switzerland: performance without competition. An example for France?

\section{Christian Desmaris}

\section{OpenEdition \\ Journals}

Édition électronique

URL : http://journals.openedition.org/pmp/7028

ISSN : 2119-4831

Éditeur

Institut de Management Public (IDPM)

\section{Édition imprimée}

Date de publication : 15 avril 2014

Pagination : 169-191

ISBN : 978-2-7430-1997-6

ISSN : 0758-1726

Référence électronique

Christian Desmaris, "La régionalisation ferroviaire en Suisse : la performance sans la compétition. Un exemple pour la France? », Politiques et management public [En ligne], Vol 31/2 | 2014, mis en ligne le 23 décembre 2016, consulté le 01 mai 2019. URL : http://journals.openedition.org/pmp/7028 


\title{
La régionalisation ferroviaire en Suisse : la performance sans la compétition. Un exemple pour la France?
}

\author{
Christian Desmaris \\ UMCF de sciences économiques, \\ Institut d'études politiques de Lyon - Laboratoire d'économie des transports (LET), \\ UMR 5593, 14 av. Berthelot, 69365 Lyon, cedex 07, France
}

\section{Résumé}

Dans un contexte de régulation renouvelée par I'Union européenne, la France a entrepris une réforme graduelle de son système de transport régional de voyageurs. Après une phase d'expérimentation, le transfert aux régions de la compétence d'organisation et de financement du service public régional de transport de voyageurs a été généralisé. Aujourd'hui, face à des finances publiques locales détériorées et à l'ouverture à la concurrence prochaine, la régionalisation ferroviaire française doit être approfondie. Comment obtenir davantage de services avec moins d'argent public? Quelle gouvernance publique établir?

Cet article se propose de formuler quelques suggestions à partir d'une analyse de la réforme du trafic ferroviaire régional conduite en Suisse, pays reconnu pour l'excellence de son système ferroviaire. Suite à cette réforme, la Suisse a enregistré d'importants gains, à la fois en termes de qualité de services offerts aux passagers et de coût payé par les contribuables. Cette performance n'est pas imputable à davantage de concurrence, mais à la nature de la gouvernance publique et à son impact sur le jeu des acteurs du système ferroviaire. Si ces gains de performance ont aussi un coût et dépendent de nombreux facteurs, parfois très spécifiques, quelques enseignements peuvent être énoncés pour la France.

C 2014 IDMP/Lavoisier SAS. Tous droits réservés

Mots clés : réforme des chemins de fer, transport ferroviaire régional de voyageurs, régulation, concurrence, Suisse.

\section{Abstract}

Regionalization railway in Switzerland : performance without competition. An example for France? In the context of a renewed regulation by the European Union,

*Auteur correspondant : christian.desmaris@sciencespo-lyon.fr doi :10.3166/pmp.31.169-191 @ 2014 IDMP/Lavoisier SAS. Tous droits réservés 
France has undertaken a gradual reform of its system of regional passenger transport. After an experimental phase, transfer to the regions of the organization and financing competence for the regional public service passenger was widespread. Today, to face the deteriorated local public finances and the next opening to competition, regionalization French rail must be thorough. How to get more services with less public money? Which public governance establish?

This article offers some suggestions from an analysis of the regional rail traffic reform driving in Switzerland, a country recognized for the excellence of its rail system. Following this reform, Switzerland has made significant gains, both in terms of quality of services offered to passengers and in terms of cost paid by taxpayers. This performance is not due to more competition, but to the nature of public governance and to its impact on the rail system actors itself. If these performance gains have also a real cost and depend on many factors, some very specific, nevertheless few lessons can be set for France. @ (c) 2014 IDMP/Lavoisier SAS. Tous droits réservés

Keywords: Railway Reform, Regional Passenger Rail, Regulation, Competition, Swiss.

\section{Introduction}

Le trafic régional de voyageurs en France a été métamorphosé depuis plus de dix ans par la réforme qui, en 2002, a introduit une régionalisation de ce service (loi 2000-1208, du 13 décembre 2000). Au-delà de nombreux succès, notamment en termes de trafic (Haenel, 2008, 2011; SETRA, 2009; Crozet, Desmaris, 2011), cette réforme apparaît aujourd'hui comme inachevée et même face à une triple impasse, à la fois financière (Cour des Comptes, 2009), institutionnelle (Assises du ferroviaire, 2011; ARF, 2011) et réglementaire (Abraham, 2011; Grignon, 2011; CESE, 2012).

Nombreux sont ceux qui attendent de l'ouverture à la concurrence, promise par les évolutions récentes de la législation européenne, à travers le règlement relatif aux services publics de transport de voyageurs par rail et par route (1370/2007/CE) et maintenant par le Quatrième paquet ferroviaire, une réponse à ces impasses. Avec le règlement européen 1370/2007, la « concurrence régulée », aussi appelée concurrence «pour le marché », deviendra la norme explicite pour l'organisation du marché des services de transport de voyageurs local ou régional. Une procédure d'appel d'offres devra systématiquement précéder l'octroi d'une licence d'exploitation par une autorité publique. Le Quatrième paquet ferroviaire généralise ce principe à tous les contrats de service public relatif au marché domestique de transport de voyageurs, à compter de décembre 2019 (COM2013/25/CE).

Dans ce contexte, l'objectif de cet article est de mettre en lumière les choix effectués et les résultats obtenus par un pays voisin, la Suisse, réputée pour l'excellence de son réseau ferroviaire. Dans ce pays, les réformes des chemins de fer et, en particulier, la régionalisation ferroviaire semble avoir produit des gains de performance significatifs sans avoir plongé le transport ferroviaire de passagers dans une concurrence ouverte « pour le marché » et encore moins « sur le marché ». L'ensemble du trafic ferroviaire régional est organisé sous forme de concessions attribuées aux Chemins de Fer Fédéraux (CFF), soit par la Confédération pour le trafic national longue distance, soit par les autorités cantonales 
pour le trafic régional et local. Nous suggérons que la qualité de la gouvernance publique est le principal facteur de progrès. Quelle est la nature de cette gouvernance ferroviaire helvétique? Peut-elle devenir un «modèle » pour la réforme prochaine des chemins de fer régionaux en France? Notre approche a reposé sur une investigation approfondie de la littérature académique, mais aussi officielle suisse, notamment réglementaire, complétée par des échanges avec quelques acteurs du système de transport public suisse ${ }^{1}$. Nous avons également eu abondamment recours aux statistiques ferroviaires nationales suisses et de l'UIC, ainsi qu'à de nombreuses publications et données de gestion des Chemins de fer fédéraux $(\mathrm{CFF})$ - Voir Annexe.

Dans une première partie, nous caractériserons les récentes réformes de l'environnement institutionnel du transport ferroviaire de passagers en Suisse et nous en présenterons une évaluation centrée sur leur impact pour le trafic régional tant en matière de finances publiques que de services aux voyageurs. Puis, dans une seconde partie, nous expliquerons les raisons des succès obtenus, pour, dans une dernière partie, dégager quelques enseignements et quelques propositions à même de contribuer à éclairer la prochaine réforme du transport ferroviaire régional en France.

\section{La réforme du transport ferroviaire de voyageurs en Suisse : originale et fructueuse}

Si les motivations qui président à la réforme ferroviaire suisse sont similaires à celles prévalant dans les autres pays européens (ECMT, 1998; OFT, 1998 ; CER, 2011), les orientations prises et son calendrier définissent, pour le trafic de voyageurs, un environnement institutionnel bien différent de celui proposé comme modèle par l'Union européenne. La concurrence pour le marché proposé par le droit communautaire pour le trafic donnant lieu à des contrats de service public, comme le trafic régional, bien que formellement introduit en Suisse par le nouveau cadre réglementaire, n'est pas sollicitée. Et pourtant de nombreux gains de performances ont été obtenus, tant en matière de coût pour les contribuables que de services pour les voyageurs.

\subsection{Un schéma institutionnel original : gouvernance versus concurrence}

Pour comprendre la nature du nouvel environnement institutionnel régissant le transport ferroviaire régional de voyageurs en Suisse, nous le replacerons dans la dynamique générale des réformes du transport ferroviaire de voyageurs de ce pays. La réforme des chemins de fer suisse peut être présentée en distinguant trois étapes : en 1996, la « Révision de la loi fédérale sur les chemins de fer » (LCDF), que nous assimilerons à un processus de « régionalisation » puis, en 1999, la « Réforme des chemins de fer 1 », plus globale, et depuis 2005, la «Réforme des chemins de fer 2 », encore partiellement en discussion et en cours de mise en œuvre (CER, 2011).

\footnotetext{
${ }^{1}$ Nous citerons en particulier divers contacts auprès de l'OFS (Office fédéral de la statistique), de spécialistes du transport ferroviaire de l'EPFL, de responsables d'autorité de transport cantonale et des CFF. Nous tenons à souligner la grande facilité d'accès que les CFF nous ont autorisé à certaines parties de leur propre base de données.
} 


\section{La première étape en 1996 : la réforme du trafic régional}

La réforme ferroviaire suisse commence par une redéfinition des principes qui régissent le transport régional de passagers. Ainsi, en Suisse, la régionalisation ferroviaire précède et initie les réformes des chemins de fer. Cette première réforme, introduite par le LCDF du 24 mars 1995 et entrée en vigueur le $1^{\text {er }}$ janvier 1996, est basée sur trois principes qui vont profondément transformer la relation entre les entreprises ferroviaires et les administrations publiques (Genoud, 2000).

Le changement le plus important est connu comme le « principe de commande ». Il signifie que les pouvoirs publics s'engagent à ne payer que pour les services convenus à l'avance (pour une ligne spécifique, dans une période de temps spécifique, et pour la fourniture de certains services déterminés) et uniquement pour le montant prédéfini par le contrat. La compensation financière est calculée à partir de l'estimation du total des coûts encourus, après déduction du chiffre d'affaires généré par le service (CER, 2005). Ainsi, l'allocation correspond uniquement au déficit prévu par le transporteur au début de la période. Les déficits imprévus ne sont plus couverts par la suite par l'État. Ce contrat, appelé « coût net », incite le concessionnaire à la fois à un meilleur contrôle de ses coûts et à augmenter ses recettes, puisque ses pertes éventuelles ne seront pas indemnisées ex post. Les entreprises de transport public assument ainsi tous les risques entrepreneuriaux de leurs activités.

La deuxième nouveauté concerne les pouvoirs dévolus aux autorités cantonales. Jusqu'alors, la Confédération fixait avec les CFF les conditions de base de l'offre de transport régional de passagers et elle en assumait la charge financière. Les cantons n'étaient associés que pour présenter leurs requêtes en matière d'horaires et ne se positionnaient qu'en tant que «quémandeurs » (Blumenthal, 1998). Avec cette réforme, les autorités cantonales deviennent pleinement responsables de l'organisation des services régionaux de transport sur leur propre territoire. Cependant, leur autonomie de décision reste supervisée par l'Office fédéral des transports (OFT) qui, garant de la coordination du trafic à l'échelle nationale, co-signe le contrat et verse directement aux opérateurs la compensation financière prévue.

La troisième innovation de la réforme ferroviaire consiste en la suppression du monopole des CFF pour les services ferroviaires régionaux ${ }^{2}$. Cette disposition, sans doute la plus innovante, ouvre la possibilité de mettre plusieurs opérateurs en concurrence pour l'attribution d'un service de transport régional. Mais pour le moment, la concurrence est inexistante, dans les transports ferroviaires locaux et régionaux. Les autorités cantonales ne sollicitent pas la procédure de concours et aucun fournisseur étranger s'est introduit dans ce marché (Finger, Genoud, 2004). Il en va différemment pour le transport routier local et régional, où les procédures d'appels d'offres sont maintenant obligatoires.

Pour être vraiment complète, cette première étape de la réforme de la Suisse devait être accompagnée d'une transformation des CFF en une société bénéficiant d'une réelle autonomie de gestion. Ce sera le principal objet de la seconde réforme ferroviaire.

\footnotetext{
${ }^{2}$ II importe de préciser qu'historiquement le système ferroviaire suisse est pluriel. À côté des CFF, l'opérateur historique national, il existe une trentaine de compagnies « privées » au réseau généralement très localisé. Seuls les BLS, dont le capital appartient majoritairement au canton de Berne, disposent d'un réseau étendu. Les CFF demeurent de loin le principal opérateur avec $82 \%$ des produits du trafic passagers et $91 \%$ du trafic exprimé en voyageurs-kilomètre (Dehornoy, 2012).
} 


\section{La seconde étape en 1999 : un nouveau cadre réglementaire harmonisé avec le droit européen}

Une étape supplémentaire dans le processus de réforme ferroviaire a été réalisée avec la « Réforme des chemins de fer 1 » (LCDF, en date du 20 mars 1998) applicable à compter du 1er janvier 1999. Son objectif prioritaire était de transposer les principes de la directive 91/440/CEE ${ }^{3}$. En premier lieu, cette réforme a introduit une séparation entre les activités de transport et de gestion de l'infrastructure. Elle a également permis le libre accès au réseau ferroviaire à tout opérateur autorisé (essentiel pour la concurrence « sur le marché ») qui est depuis lors effective pour le fret en Suisse. Enfin, elle a renouvelé de manière significative l'organisation et le modèle d'entreprise des CFF.

Une dimension majeure de cette réforme ferroviaire était d'extraire les CFF de la tutelle exercée par l'administration fédérale, afin de les établir en tant que société commerciale. Cette entreprise a ainsi obtenu son indépendance à l'égard du pouvoir politique et administratif, bien que bénéficiant depuis lors d'un statut spécial de société anonyme publique, propriété exclusive du gouvernement fédéral. Ce faisant, l'administration fédérale ne dispose plus que d'une influence limitée sur les CFF. Leur principal canal d'influence est un contrat pluriannuel qui fixe les orientations stratégiques de l'entreprise et ses objectifs à moyen terme. Ce contrat spécifie également les services offerts en matière de trafic de passagers et de marchandises et d'infrastructure ferroviaire.

En retour, ce contrat précise le montant des contributions publiques apportées à l'entreprise ferroviaire nationale. Ces douze dernières années témoignent, qu'en Suisse, les gouvernements ont en très grande partie honoré leurs engagements financiers envers les $\mathrm{CFF}$, conformément à leur contrat. Pour permettre à la société nouvellement créée de fonctionner libérée des fardeaux du passé, la Confédération l'a simultanément débarrassée de ses dettes (NERA, 2004). À l'occasion de ce changement de statut, les CFF ont également opté pour une réorganisation radicale. Les activités ont été réparties en quatre branches distinctes : les passagers, les marchandises, l'infrastructure et l'immobilier. Dans l'esprit de la réglementation européenne, les CFF ont mis en œuvre la séparation des comptes et la séparation organique entre l'infrastructure ferroviaire et l'exploitation des trains, afin d'accroître la transparence dans la fourniture des services ferroviaires. Pris ensemble, ces éléments ont offert un nouveau départ aux CFF, leur permettant d'affronter et de s'affirmer dans le marché ferroviaire européen, désormais libéralisé.

Cette réforme des CFF a eu aussi de profondes implications sur la régulation du secteur et, en particulier, sur la nature des tâches confiées à l'Office fédéral des transports. Les missions des CFF, dites de « souveraineté », en conflit avec leur nouveau statut d'entreprise ferroviaire, ont dû être transférées à l'Office fédéral des transports (OFT) ${ }^{4}$.

\section{La troisième étape de la réforme ferroviaire depuis 2005 : controversée et inaboutie}

En 2005, le gouvernement a présenté un nouveau paquet ferroviaire au Parlement dit « Réforme ferroviaire 2 ». Son objectif principal était de transposer dans le droit suisse le

\footnotetext{
${ }^{3}$ Conformément à l'accord sur les transports terrestres (1999) qui harmonise partiellement les droits du transport entre la Suisse et l'Union européenne.

${ }^{4}$ En particulier, la mission d'attribution des sillons a été confiée en 2006 à un organe indépendant des entreprises ferroviaires, Sillon Suisse SA. II vise une attribution non discriminatoire des sillons et une utilisation optimale des capacités, dans l'esprit du Premier paquet ferroviaire de l'UE. Sa forme juridique et ses compétences ne sont pas encore complément établies.
} 
Premier et le Deuxième paquet ferroviaire de l'UE. Ce paquet législatif prévoyait diverses mesures touchant à de multiples sujets, tels que la façon de financer l'infrastructure ferroviaire, la façon d'établir une autorité de réglementation capable d'assurer une répartition équitable des sillons entre toutes les sociétés ferroviaires ou encore relatif à la sécurité dans les transports publics. Le Parlement a rejeté ce paquet législatif et a proposé au gouvernement de le scinder en différents projets de loi, plus ciblés (CER, 2011). Le gouvernement a alors cherché à introduire la Réforme des chemins de fer 2, par segments.

La première partie de cette réforme, relative à la réglementation du système ferroviaire, à la sécurité et à la période de commande dans le transport régional (qui d'annuelle est devenue biannuelle), est entrée en vigueur en 2010. Cette réforme comprend en particulier la loi sur le transport de voyageurs du 20 mars 2009 dit «LTV ». Elle sera complétée par l'ordonnance sur l'indemnisation du trafic régional de voyageurs (OIRTV) du 11 novembre 2009. La deuxième partie de la Réforme des chemins de fer 2 , soumise à une consultation populaire en 2009, a été présentée au Parlement à partir de l'année 2011. Certains textes sont aujourd'hui adoptés et parfois entrés en vigueur, d'autres sont encore en cours de discussion ${ }^{5}$.

Nous observons, avec grand intérêt, que trois points de cette nouvelle phase de la réforme ferroviaire suisse sont particulièrement controversés : 1) l'importance accordée aux principes de la concurrence dans l'attribution des concessions dans le transport de passagers (surtout par rail);2) les règles relatives au financement des infrastructures et, en particulier, celles qui régissent la part respective de la Confédération et des cantons; et 3) le choix de l'architecture optimale pour la gestion des infrastructures (Litra, 2008). Le système ferroviaire suisse est globalement intégré, sur le modèle japonais ou allemand ${ }^{6}$. En Suisse, l'unicité du système ferroviaire est très généralement présentée comme une condition essentielle de ses performances exceptionnelles (Meier et ali., 2011).

Globalement, les évolutions de la législation des chemins de fer suisses expriment une réforme pragmatique, étape par étape, qui vise, au moins, quatre objectifs (OFT, 1998) : premièrement, inscrire la Suisse dans le nouveau paysage ferroviaire européen; ensuite, accroître l'efficacité du transport ferroviaire, en augmentant considérablement la productivité et la rentabilité de l'exploitation, la qualité et la quantité des services offerts. Elle vise aussi à relever les défis posés par l'accroissement de la mobilité, en augmentant la part du rail. Elle prétend enfin améliorer le rapport coûts / avantages des subventions publiques.

Si le principe de la concurrence est accepté, pour le trafic fret, on ne peut manquer d'observer que le « modèle ferroviaire suisse » diffère de la norme promue par la Commission européenne, fondée sur la libéralisation du marché et sur la concurrence entre les opérateurs. L'absence d'ouverture à la concurrence pour le trafic domestique de voyageurs (régional et national), l'absence de gestionnaire d'infrastructure indépendant ou encore de véritable régulateur sectoriel font de l'architecture ferroviaire suisse un système « hybride » entre libéralisation et intégration (Maier-Gyomlay, 2013), partiellement conforme au droit communautaire et à sa philosophie (Meyer et Meier, 2011). Le modèle de la régionalisation/

\footnotetext{
${ }^{5}$ Pour une brève présentation de la situation législative actuelle, voir le site du DETEC : http://www.uvek. admin.ch/dokumentation/00474/00492/index.html?lang=fr\&msg-id=27626

${ }^{6}$ Cette intégration signifie que chaque entreprise ferroviaire est aussi responsable des infrastructures qu'elle utilise. Cette intégration verticale s'accompagne d'une multiplicité d'opérateurs ferroviaires dont les circulations s'interpénètrent. Les opérateurs intégrés ont ainsi en Suisse déjà une pratique courante de l'accès de tiers à leur infrastructure.
} 
réforme ferroviaire suisse est donc spécifique sur le plan institutionnel (Finger et Holterman, 2013), mais non stabilisé; toutes ses contradictions internes n'étant pas résolues (MaierGyomlay, 2013).

Quels en ont été les résultats, tant pour les contribuables que pour les voyageurs? Des gains de performance ont-ils été au rendez-vous? Les objectifs poursuivis ont-ils été atteints?

\subsection{Une tendance à l'amélioration des performances sans concurrence directe}

Deux résultats sont à mettre à l'actif de la réforme ferroviaire suisse du transport régional de passagers : la progression de l'efficience dans l'utilisation des fonds publics et l'accroissement de la qualité et de l'offre de services pour les passagers du rail.

\section{Plus d'efficience dans l'utilisation des fonds publics}

Le résultat de la réforme le plus spectaculaire, surtout en comparaison avec la France, est l'inversion de la tendance à l'augmentation continue des indemnités publiques. Dans les années 1990, le coût public du transport régional s'alourdissait fortement, passant de 560 millions de CHF en 1990 à 725 millions de CHF en 1993 (chiffres exprimés en valeur courante). Depuis la réforme, les subventions versées par la Confédération au titre du trafic régional ont diminué, pour s'établir à 546 millions de CHF en 2000 et même à 507 millions de CHF en 2002, au minimum (selon les chiffres des CFF). Depuis lors, ces subventions ont à nouveau augmenté, mais bien faiblement et, en fait, l'efficience des indemnités publiques s'est considérablement améliorée. Pour un train-kilomètre offert, le gouvernement suisse devait payer 10,2 CHF en 2000, pour seulement 7,6 CHF en 2006 (tout comme en 2010) et légèrement plus en 2011, avec 7,7 CHF.

Ainsi, l'amélioration de l'efficience des indemnités publiques versées aux CFF au titre du transport ferroviaire régional est spectaculaire, plus de $25 \%$ depuis 2000 (tableau 1 données non disponibles avant).

Tableau 1 : Contributions publiques compensatoires versées aux CFF au titre du transport régional de voyageurs

\begin{tabular}{|c|c|c|c|c|c|c|c|c|c|}
\hline & 2000 & 2002 & 2004 & 2006 & 2007 & 2008 & 2009 & 2010 & 2011 \\
\hline $\begin{array}{l}\text { En millions de CHF } \\
\text { courants }\end{array}$ & 546 & 507 & 522 & 552 & 592 & 571 & 559 & 556 & 577 \\
\hline $\begin{array}{l}\text { Indice, base } 100 \text { : } \\
2000\end{array}$ & 100,0 & 92,9 & 95,7 & 101,2 & 108,4 & 104,6 & 102,4 & 101,9 & 105,4 \\
\hline $\begin{array}{l}\text { CHF courants par } \\
\text { train-km }\end{array}$ & 10,19 & 8,89 & 8,11 & 7,59 & 7,71 & 8,07 & 7,90 & 7,60 & 7,70 \\
\hline $\begin{array}{l}\text { Par train-km - } \\
\text { Indice, base } 100 \\
\text { en } 2002\end{array}$ & 111,7 & 100,0 & 91,2 & 85,4 & 86,7 & 90,8 & 88,9 & 85,5 & 86,6 \\
\hline
\end{tabular}

Source : Nos calculs à partir de divers Rapports de gestion des CFF.

La comparaison avec la France souligne une grande divergence en matière d'efficacité des subventions publiques à destination des chemins de fer régionaux. Depuis la réforme en 2002, les contributions publiques pour les services ferroviaires voyageurs régionaux n'ont 
cessé d'augmenter, passant de 1405 millions d'euros en 2002 à 2572 millions d'euros en 2011 . Ceci signifie une hausse de $83 \%$ au cours de cette période, en valeur courante. Pour un trainkilomètre, en France, la Collectivité a dû payer de plus en plus : 9,45 $€$ en 2002, 11,90 $€$ en 2006 et 15,20 € en 2011 (basé sur les Comptes des transports de la nation). Calculée depuis 2002, la détérioration de l'efficacité des fonds publics accordés à la SNCF et à RFF pour l'exploitation du transport ferroviaire régional est spectaculaire, de plus de $60 \%$ (tableau 2$)^{7}$.

Tableau 2 : Contributions publiques compensatoires versées à la SNCF au titre du transport régional de voyageurs

\begin{tabular}{lcccccccc}
\hline & 2002 & 2004 & 2006 & 2007 & 2008 & 2009 & 2010 & 2011 \\
\hline $\begin{array}{l}\text { En millions d'euros } \\
\text { courants (a) }\end{array}$ & 1405 & 1468 & 1764 & 2056 & 2224 & 2325 & 2444 & 2572 \\
Indice, base 100 : 2002 & 100,0 & 104,5 & 138,0 & 146,4 & 158,3 & 165,5 & 174,0 & 183,1 \\
$\begin{array}{l}\text { Euros courants par } \\
\text { train-km }\end{array}$ & 9,45 & 11,18 & 11,91 & 12,59 & 12,90 & 13,29 & 14,52 & 15,19 \\
\hline $\begin{array}{l}\text { Par train -km Indice, } \\
\text { base 100 en 2002 }\end{array}$ & 100,0 & 118,3 & 126,0 & 133,2 & 136,5 & 140,6 & 153,7 & 160,8 \\
\hline
\end{tabular}

Source : Nos calculs à partir de divers Comptes des Transports de la nation, MEEDDAT/SESP.

(a) Les indemnités compensatoires liées au trafic régional de voyageurs comprennent les contributions publiques versées par les régions à la SNCF au titre de l'exploitation des services par la $\mathrm{SNCF}$, les compensations pour tarifs sociaux et la couverture des péages RFF. Elles n'intègrent pas la participation financière des régions au titre de l'investissement en matériel roulant, ou celle pour la rénovation ou la construction de gares ou de l'infrastructure.

Notre première conclusion est, qu'en Suisse, le grand bénéficiaire de la réforme des chemins de fer est la Confédération (et au final, le contribuable). Ce n'est pas le cas en France où les coûts en subventions publiques ont augmenté de façon spectaculaire.

\section{Plus de qualité de services et plus d'offre pour les passagers du rail}

Le deuxième résultat de la réforme ferroviaire, particulièrement enviable, consiste en une amélioration significative de la qualité de service aux passagers du rail. La Suisse est traditionnellement connue comme le pays de l'excellence ferroviaire (Bovy, 1992; Pflieger, 2008; Duranton et alii., 2012). L'intermodalité atteint un niveau inégalé ailleurs, reliant tous les éléments constitutifs du réseau de transport public suisse (Bovy, 1994; UTP, 2008). La fréquence des dessertes est la plus élevée du monde, plaçant la Suisse devant le Japon, avec plus de 94 trains par jour et par lignes (en comparaison avec 47 en Allemagne, 40 en Grande-Bretagne et seulement 30 en France - Litra, 2008). La ponctualité des circulations demeure à un niveau exceptionnellement élevé, proche de $90 \%$ en 2011 (pourcentage de passagers qui arrivent à l'heure avec moins de 3 minutes de retard), avec un taux de correspondances réussies avoisinant les $97 \%$ (CFF, 2012a).

\footnotetext{
${ }^{7}$ Selon la Cour des Comptes (2009), cette dégradation résulte pour l'essentiel de l'insuffisante maîtrise par la SNCF de ses charges d'exploitation et du choix fait par les Régions de faire supporter cette augmentation des coûts en sollicitant davantage le contribuable que l'usager.
} 
La réforme des chemins de fer a conduit à deux autres résultats attrayants du point de vue du voyageur : l'augmentation de la vitesse commerciale et la croissance de l'offre de trains.

Tableau 3 : Distances, durées et vitesses des déplacements selon le micro-recensement

\begin{tabular}{lccccc}
\hline & & 1994 & 2000 & 2005 & 2010 \\
\hline Distance par jour en km & Voiture & 21,3 & 23,6 & 23,7 & 23,8 \\
& Train & 4,2 & 4,7 & 5,6 & 7,1 \\
Durée par jour en minutes & Voiture & 32,0 & 35,3 & 34,6 & 33,2 \\
& Train & 4,6 & 4,9 & 5,2 & 6,4 \\
Vitesse en km/heure & Voiture & 37,0 & 35,5 & 36,2 & 38,6 \\
& Train & 49,8 & 53,5 & 60,9 & 61,4 \\
\hline
\end{tabular}

Source : OFS (2012), La mobilité en Suisse - Résultats du Micro-recensement mobilité et transports 2010, p. 89.

L'efficacité du transport ferroviaire de voyageurs exprimée en termes de gain de temps s'est accrue, davantage que celle de la route : la vitesse moyenne des voyages en trains a augmenté de façon substantielle, de plus de $23 \%$, passant de moins de $50 \mathrm{~km} /$ heure en moyenne en 1994 à plus de 61 km/heure en 2010. La vitesse des parcours en voiture est restée bien inférieure, autour de 37-38 km/heure, ne progressant quasiment pas (tableau 3). L'essentiel de ce gain de vitesse a été obtenu entre 1994 et 2005, consécutivement à la mise en œuvre du vaste programme d'investissement et de modernisation appelé « Rail 2000 »(Carron, 2004; Weibel, 2012). Rail 2000 a significativement amélioré l'attractivité du rail pour les passagers, en généralisant le cadencement (au quart d'heure sur les lignes les plus fréquentées et à la demi-heure pour les autres) et en systématisant les correspondances entre le rail et les autres modes de transport (Torrego, 2012). Cette meilleure performance du transport ferroviaire s'est aussi accompagnée d'un effet collatéral avantageux, un développement de l'usage du train, en comparaison avec la voiture. Alors que la distance journalière moyenne en train était de 4,2 km en 1994, elle est de 7,1 km en 2010, soit une hausse de $67 \%$ (contre $12 \%$ pour la voiture).

Dernier facteur d'amélioration de la qualité, mais non des moindres, l'accroissement de l'offre de services. Mesurée en trains-kilomètres, l'offre totale des CFF pour les voyageurs est passée de 105 millions en 2002 à 141,8 millions en 2011. Cela représente une augmentation de $35 \%$ au cours de la période 2002-2011, soit une hausse annuelle moyenne de 3,4 \%. Pour le seul transport régional (y compris par bus), l'offre kilométrique est passée de 56,8 millions de trains-kilomètres à 74 millions, soit un accroissement de $30 \%$ au total ou une progression de 3,0 \% en moyenne annuelle (CFF, 2012a). Au cours de cette même période, l'offre de service de transport régional en France a augmenté un peu plus lentement, de 20,6\%,passant de 148,7 millions de trains-kilomètres à 179,3 millions, soit une hausse de 2,1\% par an en moyenne (Crozet, Desmaris, 2011). Sur cet indicateur, la Suisse est encore meilleure que la France.

Il importe maintenant d'expliciter les facteurs au cœur de ces performances ferroviaires.

\section{Les leviers de la réussite : un jeu d'acteurs « gagnant-gagnant » impulsé par les pouvoirs publics}

Selon nous, les gains de performances ferroviaires obtenus en Suisse sont principalement redevables de la nature de la gouvernance publique qui tend à fédérer l'ensemble des 
acteurs du système ferroviaire, à savoir les pouvoirs publics, les opérateurs ferroviaires et la population, dans son rôle de clients et de citoyens-contribuables.

\subsection{La première clé du succès : une gouvernance publique en faveur d'une mobilité soutenable centrée sur le système ferroviaire}

La raison principale des succès de la réforme ferroviaire suisse tient à une gouvernance publique très spécifique qui vise à promouvoir un système de mobilité durable centré sur le rail. Nous en éclairerons trois caractéristiques.

\section{Un choix collectif en faveur du transport ferroviaire basé sur un niveau élevé d'investisse- ment dans les infrastructures}

La Suisse se distingue par un choix collectif affirmé et renouvelé en faveur du transport ferroviaire, qui se traduit par une continuité investissements importants (ECMT, 1999; Keseljevitch et ali., 2001; Carron, 2004). Après avoir donné la priorité à la route, la politique suisse des transports connaît une rupture en faveur du rail à partir des années 1990. Suite à d'intenses débats publics et consultations populaires, un vaste programme de modernisation du réseau ferré est adopté en 1998. Ce programme, d'un montant de plus de 30 milliards de francs à répartir sur 20 ans, est appelé localement GPF (grands projets ferroviaires). Il donne la priorité à une profonde modernisation du transport ferroviaire de passagers, placé au centre du réseau de transport public. Pour être en mesure de transporter des marchandises de manière plus écologique en Suisse, il comprend également un ambitieux programme de nouvelles lignes ferroviaires alpines, les NLFA (OECD, 2006).

Deux chiffres illustrent la nette priorité donnée depuis lors au transport ferroviaire en Suisse. En 2009, le gouvernement fédéral a investi 4,5 milliards de francs suisses pour la route contre 3,8 milliards de CHF dans le rail (Litra, 2011a). En 1990, le rapport était de 3,7 milliards de francs suisses pour la route contre à peine 2 milliards pour le rail. Ce choix collectif se reflète également dans le niveau des investissements ferroviaires par habitant ${ }^{8}$ : 82 CHF en 1980, 291 CHF en 1990, 405 CHF en 2000, 600 CHF en 2005 et 489 CHF en 2009, soit une multiplication par près de 6 en moins de 30 ans en valeur courante. Par comparaison, les investissements dans l'infrastructure ferroviaire en France ont aussi augmenté récemment (CGDD, 2011), mais nettement moins qu'en Suisse : ils sont passés, en équivalent francs suisses, de 31,9 CHF par habitant en 2000 à 61,4 CHF en $2009^{\circ}$.

\section{Une implication croissante des collectivités territoriales dans la décision et le financement}

La réforme des chemins de fer suisses comprend un processus de décentralisation qui se manifeste de deux manières : une pleine responsabilité des cantons dans la décision en matière de services, notamment pour la consistance de l'offre, d'une part, et, en retour, une prise en charge de ce service par les finances publiques locales. Le contrat est librement négocié entre les deux parties, à savoir le canton et l'entreprise ferroviaire. Dans la pratique,

\footnotetext{
${ }^{8}$ Ces investissements incluent les dépenses dans les installations et les équipements, ainsi que celles pour le renouvellement et l'entretien du secteur des constructions (Litra, 2011a).

${ }^{9}$ Les conversions sont faites sur la base de 1 franc suisse pour 0,66 euro en 200 et de 1 CHF pour 0,66 euro en 2009 (INSEE).
} 
l'entreprise ferroviaire soumet au canton une offre pour les services publics demandés. Si le prix de l'indemnité compensatrice est librement négocié entre les parties, le contrat doit être approuvé par l'OFT (CER, 2005).

Depuis 2004, les relations financières entre la Confédération et les cantons sont régies par de nouvelles règles, la nouvelle péréquation financière (NPF). Ce cadre financier a conduit à rendre plus difficile l'accès aux fonds fédéraux et à borner la part assumée par la Confédération en matière de financement du trafic régional de voyageurs (art. 6 de l'OIRTV du 11 novembre 2009). La part de la Confédération qui était encore en 2003, en moyenne nationale, de $65 \%$ des contributions versées au titre du trafic régional (OFS, 2005) a rejoint le seuil de $50 \%$ prévu par la loi (OFS, 2010). À la fin de l'année 2010, le Conseil fédéral a tenté de restreindre davantage sa contribution, en proposant d'augmenter le seuil de trafic minimal ouvrant droit à l'indemnisation du transport régional, le passant de 32 à 100 passagers par ligne et par jour. Aujourd'hui, le seuil minimal de couverture des frais par les recettes, qui conditionne la participation financière de la Confédération, est de $20 \%$ (art. 4 de la Directive TRV du 10 décembre 2010).

\section{Une gouvernance d'entreprise des CFF vraiment incitative et responsabilisante}

La profonde transformation des relations entre les CFF et leur actionnaire, la Confédération, semble être un facteur clé, trop rarement souligné, des succès rencontrés par la réforme ferroviaire suisse. Deux aspects de la gouvernance des CFF par les pouvoirs publics helvétiques méritent d'être soulignés : la fixation d'objectifs stratégiques clairs, précis et strictement contrôlés et l'imposition d'une contrainte financière drastique à l'opérateur ferroviaire.

Le premier terme de cette gouvernance d'entreprise réside dans le véritable pilotage stratégique de la compagnie ferroviaire nationale par son actionnaire. L'activité des CFF est inscrite dans un contrat à moyen terme avec son propriétaire, appelée «Convention sur les prestations ». Chaque convention exprime finement les objectifs politiques et stratégiques des CFF, y compris les services publics commandés par la Collectivité pour les quatre années à venir. La convention prévoit, pour la même période de temps, un plafond pour les crédits accordés tant pour l'infrastructure que pour les services d'exploitation. Le processus de contrôle de la société par ses superviseurs est également clairement indiqué.

En Suisse, le politique, le Conseil fédéral, définit explicitement les objectifs stratégiques qu'il attribue aux CFF. Bien que rédigés conjointement avec la société, ces objectifs souscrivent à une séparation claire des pouvoirs entre le propriétaire et l'exploitant, principe requis par la réforme. Ce cadre stratégique permet aux CFF de prendre librement les décisions entrepreneuriales appropriées. On note combien ces objectifs visent à obtenir une amélioration constante de la qualité de services et des gains d'efficience dans l'utilisation des fonds publics. La satisfaction du client, la ponctualité et la sécurité et, plus généralement, un haut niveau de service doivent s'accompagner de gains de productivité systématiques. Des résultats financiers fournissant une base saine pour toute l'entreprise et un résultat équilibré, à moyen terme, dans chaque domaine sont également attendus.

La Collectivité détermine expressément les ressources financières allouées. Concernant le transport régional, les subsides sont spécifiés dans le cadre de la procédure de commande, par une convention sur les indemnités. Nous avons vu comment cette innovation contractuelle a significativement contribué à la maîtrise des contributions compensatoires versées aux $\mathrm{CFF}$ au titre du trafic régional. Ce dispositif présente un double mérite. Il est, 
d'une part, particulièrement incitatif pour l'opérateur, qui ne peut plus compter sur des fonds publics en cas de dérive de ses comptes. Symétriquement, il autorise une visibilité financière à moyen terme pour les pouvoirs publics. La contrepartie de cet équilibre est de devoir travailler dans un cadre contractualisé et donc soumis à évaluation.

Quant au trafic longue distance, suite à la réforme, il doit être autonome sur le plan financier. Les recettes générées par les droits exclusifs, accordés par les pouvoirs publics, doivent être suffisantes pour couvrir les coûts d'exploitation de l'entreprise ferroviaire sur ce trafic.

Les activités des CFF donnent lieu à un contrôle vigilant, transparent et permanent exercé tant par la Direction de l'entreprise que par son actionnaire. Nous sommes frappés par la finesse du reporting périodique (mensuel et annuel) prévu par la Convention à partir d'une batterie d'indicateurs reposant parfois sur des valeurs-seuils valant objectif. Les rapports annuels de gestion des CFF sont à la fois précis, exhaustifs ${ }^{10}$, argumentés et, semble t-il, sincères.

Le deuxième terme de cette gouvernance d'entreprise originale, initiée par les pouvoirs publics, s'est traduit par une contrainte financière absolue imposée par la Confédération aux CFF. La stratégie explicite de la Confédération, maintenue sur la durée, convention après convention, est de stabiliser le total des fonds publics alloués aux CFF, tout en accroissant les exigences en termes de résultats à atteindre (tableau 4). La logique ici est clairement de «faire plus avec moins de moyens ».

Tableau 4 : Évolution du plafond de dépenses au profit des CFF

\begin{tabular}{ccc}
\hline Conventions sur les prestations & $\begin{array}{c}\text { Montant global } \\
\text { (millions de CHF) }\end{array}$ & $\begin{array}{c}\text { Moyenne annuelle } \\
\text { (millions de CHF) }\end{array}$ \\
\hline $1999-2002$ & 5840 & 1460 \\
$2003-2007$ & $6020(5602)(\mathrm{a})$ & $1505(1400)(\mathrm{a})$ \\
$2007-2010$ & 5880 & 1470 \\
$2011-2012$ & 3322 & 1661 \\
$2013-2016$ & 6624 & 1656 \\
\hline
\end{tabular}

Source : Litra (2011b), Doku; Convention sur les prestations entre la Confédération et les CFF 2011-2012 et 2013-2016.

(a) Après réduction suite à des programmes d'économies.

Face à ce cadre budgétaire de plus en plus exigeant, les CFF ont été tenus à des mesures drastiques et systématiques d'économies et à la recherche tous azimuts de recettes nouvelles. Cette priorité se trouve renforcée par la progression des attentes de la Confédération en termes de missions à réaliser et par la priorité absolue donnée au maintien de la qualité de l'infrastructure.

Enfin, la grande qualité de la gouvernance du système ferroviaire suisse tient probablement à un autre facteur relevé par E. Calthrop $($ CER, 2005) : l'exceptionnelle stabilité des responsables en place, tant du côté du Ministère des transports que de la Direction des CFF.

\footnotetext{
${ }^{10}$ Nous suggérons au lecteur de comparer les rapports d'activité et les rapports financiers des CFF et de la SNCF. Difficile dans le cas de la SNCF d'avoir une vision longue des variables présentées ou des détails opérationnels sur chaque segment de l'activité.
} 


\subsection{La seconde clé du succès : un opérateur historique capable de profondes innova- tions organisationnelles et de considérables gains de productivité}

Les réformes des chemins de fer en Suisse ont rapidement conduit à un bouleversement de la nature et des finalités de la gestion des CFF. Au-delà du changement de statut juridique, qui a induit une autonomie et une responsabilisation plus grande, la réforme s'est accompagnée d'une réorientation stratégique mettant la recherche de l'efficience productive au cœur des pratiques opérationnelles et les clients au centre de l'activité (Genoud, 2000).

Suite aux réformes ferroviaires, la stratégie des CFF s'est inscrite sur deux axes. Le premier axe vise à augmenter l'efficience productive, par d'intenses gains de productivité du travail et par une politique systématique de maîtrise des coûts opérationnels. Le second axe valorise la recherche tous azimuts de recettes nouvelles, tant dans le domaine du transport ferroviaire que par la valorisation des actifs patrimoniaux de la firme.

\section{Rechercher et obtenir de significatifs gains de productivité du travail}

Les CFF doivent croître tout en réduisant leurs coûts. La quête d'efficience productive des CFF se concrétise par la recherche permanente d'importants gains de productivité. Mesurés par la productivité apparente du travail, les gains obtenus par la firme sont considérables : de $92 \%$ entre 1996, le début de la réforme ferroviaire, et 2011. Cela représente une hausse de l'efficience productive au rythme soutenu de 5,1\% par an en moyenne entre 1996 et 2011 (tableau 5).

À la différence de nombreuses autres compagnies ferroviaires européennes, qui ont très largement réduit leurs effectifs (Desmaris, 2010), cette performance repose en Suisse principalement sur un développement sensible du trafic, de voyageurs et de fret $(+52 \%)$ et, dans une moindre mesure, sur une contraction des effectifs $(-21 \%)$.

Tableau 5 : Évolution du trafic, des effectifs et de la productivité des CFF

\begin{tabular}{lcccccccc}
\hline & 1996 & 1998 & 2000 & 2005 & 2009 & 2010 & 2011 & $\begin{array}{c}\text { Variation } \\
\text { en } \% \\
1996-2011\end{array}$ \\
\hline $\begin{array}{l}\text { Voyageurs-km } \\
\text { en millions (1) }\end{array}$ & 11662 & 12485 & 12835 & 13830 & 16677 & 17513 & 17749 & $+52,2$ \\
$\begin{array}{l}\text { Tonnes-km en } \\
\text { millions (2) }\end{array}$ & 8108 & 8738 & 10658 & 8571 & 11674 & 13111 & 12346 & $+52,3$ \\
$\begin{array}{l}\text { Personnel (3) } \\
\begin{array}{l}\text { Productivité } \\
\text { du travail }\end{array}\end{array}$ & 32581 & 30862 & 28272 & 25943 & 25287 & 25356 & 25840 & $-20,7$ \\
$\begin{array}{l}\text { en millions } \\
\text { d'unités } \\
\text { de trafic (4) }\end{array}$ & 0,61 & 0,69 & 0,83 & 0,86 & 1,12 & 1,21 & 1,16 & - \\
\hline $\begin{array}{l}\text { Productivité, } \\
\text { base 100 } \\
\text { en 1996 }\end{array}$ & 100,0 & 118,0 & 136,9 & 142,3 & 185,4 & 199,0 & 191,9 & $+91,9$ \\
\hline
\end{tabular}

Source : Nos calculs à partir des Statistiques chronologiques des chemins de fer 1970-2005 (UIC, 2007), de la Statistique Internationale des Chemins de Fer 2010 (UIC, 2011) et CFF (2012a).

$N B:(4)=[(1)+(2)] /(3)$. Nous supposons qu'un voyageur-kilomètre équivaut à une tonne-kilomètre. 
Cette tendance à la hausse de la productivité du travail n'est pas le fruit du hasard. Elle résulte de la politique systématique de recherche d'efficacité et de productivité engagée par les CFF dans tous les domaines (Litra, 2011c), dans le but de répondre aux contraintes de financement et aux objectifs imposés par la Confédération, son actionnaire ${ }^{11}$. Nous prendrons plusieurs exemples pour illustrer le caractère systématique de cette stratégie visant à réduire les coûts opérationnels.

Dans le domaine du transport de voyageurs, des économies de personnel ont été obtenues de plusieurs manières. La suppression du contrôle à bord des billets en est un exemple (Blumenthal, 1998). Introduite dès 1994 et généralisée à compter de juin 1996, cette mesure qualifiée « d'autocontrôle » s'accompagna d'un système nouveau de contrôle ponctuel des billets. Cette mesure se traduisit aussi par la suppression de la possibilité d'acheter son billet à bord et par la montée d'un sentiment d'insécurité. Pour pallier ces critiques, les CFF généralisèrent les automates en gare et introduisirent une police ferroviaire et des équipes de médiation pour assurer la sécurité à bord. Certaines petites gares ou haltes ont aussi été supprimées ou ont vu leurs horaires d'ouverture réduits.

Plus récemment, pour faire face aux plans d'austérité de la Confédération, qui priva l'unité Trafic régional des CFF d'une partie des subventions attendues, les CFF adoptèrent une riposte par le développement d'une stratégie de partenariat, avec des compagnies ferroviaires privées bien implantées au niveau local. Les doublons dans les secteurs de l'entretien, du marketing, de la gestion et de la vente furent réduits, avec pour conséquence un accroissement de la productivité, sans nuire pour autant à la performance du service.

Cette recherche constante de gains de productivité et d'économies donne néanmoins lieu à des critiques. Certains (Nahrath et alii., 2008) redoutent les effets sur la durabilité du système ferroviaire, tant d'un point de vue technique (le désinvestissement limite le développement futur du trafic et la robustesse de l'exploitation), que social (diverses économies compliquent l'usage du service pour certaines catégories de populations).

\section{Augmenter les revenus de la compagnie ferroviaire}

Deuxième axe des mutations managériales, impulsée par la réforme ferroviaire, placer les clients au centre de l'activité des CFF. Dans une optique « gagnant-gagnant », cette stratégie ambitionne à la fois d'améliorer la qualité du service produit et perçu par la clientèle et d'augmenter les recettes de la compagnie ferroviaire. Les résultats sont au rendez-vous.

Une lecture du compte d'exploitation des CFF à dix ans d'intervalle confirme les effets de cette stratégie sur les recettes (CFF, 2012a). Ainsi, entre l'exercice 2002 et celui de 2011, alors que les recettes de trafics voyageurs ont augmenté de $52 \%$, le total des concours des pouvoirs publics aux CFF n'a progressé que de 19,7\%. Le total des charges sur cette période a enregistré une hausse de $22,2 \%$ (et de seulement $17,6 \%$ pour la masse salariale), plus faible que celle des produits d'exploitation. Il en a résulté une amélioration du résultat d'exploitation, qui est passé de 194 millions de CHF à 350 millions en valeur courante sur cette même période.

11 Nous observerons aussi combien la hausse des trafics, facteur clé de cette augmentation de la productivité, doit certainement aussi beaucoup aux politiques publiques «volontaristes » menées en Suisse et, notamment à « Rail 2000 » (investissements dans le réseau) ou encore à la politique de report modal du fret. 
Nous observons comme dernier facteur de la stratégie des CFF, une politique particulièrement active de valorisation de ses actifs immobiliers centrée autour des gares. Le groupe CFF est en effet un des acteurs majeurs du marché immobilier en Suisse, avec 800 gares, 3500 immeubles, 4000 terrains, d'une valeur comptable de près de 4 milliards de francs suisses pour un chiffre d'affaires annuel de plus de 600 millions de francs en 2011.

En 2003, les CFF ont dissocié leur portefeuille immobilier de l'Infrastructure pour en faire une unité d'affaires autonome au sein des CFF. Gérée comme un des pôles de développement particulièrement prometteurs, cette nouvelle unité d'affaires apporte désormais une contribution essentielle, tant au succès de la politique de mobilité intégrée et à l'offre de prestations destinées à la clientèle des CFF, qu'à l'équilibre financier global de la compagnie ferroviaire.

Le bénéfice réalisé par «Immobilier » participe chaque année au paiement des intérêts et des mensualités de remboursement du prêt accordé par la Confédération pour l'assainissement de la Caisse de pensions des CFF (182,5 millions en 2011). Ce bénéfice est également mis à contribution pour financer la branche Infrastructure (pour plus de 150 millions en 2011), réduisant d'autant la contribution des pouvoirs publics à l'équilibre de cette activité. Ces prélèvements internes, effectués sur le résultat d'Immobilier, en minorent le caractère bénéficiaire et son importance stratégique réelle pour les CFF (tableau 6).

Tableau 6 : Résultats du groupe CFF par segment d'activité (en millions de CHF)

\begin{tabular}{lrrrrrrr}
\hline & 2002 & 2003 & 2004 & 2005 & 2006 & 2008 & 2011 \\
\hline Trafic de voyageurs & 113,7 & 93,4 & 152,2 & 78,6 & 193,7 & 276,8 & 213,9 \\
Trafic marchandises & $-96,1$ & $-33,1$ & $-2,8$ & $-165,7$ & $-37,3$ & $-29,9$ & $-45,9$ \\
Infrastructure & 106,5 & 0,3 & 43,7 & 17,4 & 91,8 & 30,4 & 72,4 \\
Immobilier & - & $-4,6$ & 15,2 & 21,0 & 27,8 & 3,3 & 2,4 \\
Immobilier avant versement & - & 152,1 & 184,6 & 219,6 & 229,8 & 291,6 & 182,5 \\
d'indemnités (a) & & & & & & & \\
Unités centrales (b) & $-136,4$ & $-34,3$ & $-164,2$ & $-123,2$ & $-20,5$ & 68,8 & 96 \\
Élimination intersociétés & - & 3,2 & $-1,4$ & 5,6 & 4 & $-4,6$ & 0 \\
\hline Bénéfice du groupe & $\mathbf{- 1 2 , 0}$ & $\mathbf{2 4 , 9}$ & $\mathbf{4 2 , 6}$ & $\mathbf{- 1 6 6 , 3}$ & $\mathbf{2 5 9 , 4}$ & $\mathbf{3 4 5 , 0}$ & $\mathbf{3 3 8 , 7}$ \\
\hline
\end{tabular}

Source : Nos calculs d'après divers Rapports de gestion des CFF.

(a) Après paiement des intérêts et mensualités de remboursement du prêt accordé pour l'assainissement de la Caisse de pensions CFF et versements des indemnités compensatoires à Infrastructure. Rajouté par nous pour comparaison. Ne pas prendre en compte dans le calcul du total du tableau, «Bénéfice du groupe ».

(b) Les unités centrales ont en charge les fonctions transversales au profit de l'ensemble des autres unités du groupe $\mathrm{CFF}$, telles que la fonction financière, de gestion des ressources humaines, informatique et de communication interne et externe.

Ainsi, face à un contexte exigeant, les CFF se sont révélés particulièrement innovants et efficaces. Ils ont plutôt correctement maîtrisé l'évolution de leurs coûts de production, notamment 
par l'obtention de spectaculaires gains de productivité du travail. Appuyés par des politiques volontaristes en faveur du rail, ils ont également su dynamiser leurs recettes, tant dans le domaine du transport ferroviaire que par une stratégie active, à la japonaise, de valorisation patrimoniale centrée sur le potentiel des gares. Au-delà, la réforme ferroviaire suisse n'aurait pas atteint ses objectifs si elle n'avait pas rencontré un écho favorable de la part de la population.

\subsection{La troisième clé du succès : le soutien des consommateurs et des citoyens}

Après soixante ans de progression continue de la voiture particulière, les transports publics regagnent du terrain depuis une dizaine d'années, en grande partie du fait des parts de marché voyageurs reprises par le rail.

\section{Une progression exceptionnellement soutenue du trafic de voyageurs}

La régionalisation ferroviaire marque le point de départ d'un renouveau de l'engouement des Suisses à l'égard de leur chemin de fer. Les statistiques de fréquentation sont sans appel. Le nombre de voyageurs transportés, qui stagnait à 260 millions environ au cours de la première moitié des années 2000, est passé à 274 millions en 2005 et à 357 millions en 2011 . Ainsi entre 1996 et 2011, l'augmentation du trafic de voyageurs a été de 39,1\%, soit une progression annuelle moyenne de 2,2 \%. Rail 2000 est un véritable succès (Leuenberg, 2010; Weibel, 2012). Une approche en termes de voyageurs-kilomètre confirme les effets positifs de la réforme ferroviaire suisse. De 1996 et 2011, le nombre de passagers-kilomètre est passé de 11600 millions à 17750 millions; ce qui représente une hausse totale de 52,2\% ou un taux annuel moyen de 2,8 \%. Une analyse plus fine par type de trafic indique une progression similaire du trafic régional au regard de l'ensemble du trafic domestique de voyageurs en Suisse (respectivement 44, $2 \%$ contre 45,4 \%). Le trafic régional représente une part stable de $25 \%$ du trafic total de voyageurs des CFF exprimé en passagers-kilomètre.

Cette progression du trafic de voyageurs s'avère être la plus soutenue d'Europe, après celle du Royaume-Uni, alors que la Suisse est déjà, et de très loin, le pays où l'usage du train est le plus répandu au monde. En 2010, avec une moyenne de $2258 \mathrm{~km}$ par habitant, la Suisse se positionne même devant le Japon, l'autre pays de l'excellence ferroviaire $(1910 \mathrm{~km})$, et surtout loin devant la France $(1320 \mathrm{~km})$, l'Allemagne $(961 \mathrm{~km})$ ou le Royaume-Uni $(887 \mathrm{~km})$ (Litra, 2011d). Plus encore, la Suisse a même conforté sa position de leader dans l'usage du rail, puisqu'au début des réformes, les Suisses parcouraient « seulement » $1670 \mathrm{~km}$ en train chaque année. Aucun pays n'a connu une telle progression. Ce résultat est d'autant plus remarquable, qu'en Suisse, à la différence de la France où l'essentiel des parcours se font sur de longues distances (TGV), le trafic extrêmement élevé est d'abord le fait d'une utilisation fréquente du ferroviaire, mais pour de courtes distances. Ainsi, alors qu'un Français prend en moyenne 17 fois le train par habitant et par an, le Suisse 50, l'Allemand 24 et l'Espagnol 12 (Litra, 2011d).

\section{Satisfaction des voyageurs et adhésion citoyenne}

La satisfaction exprimée par les voyageurs et l'adhésion citoyenne aux réformes ferroviaires conduites par la Confédération sont des éléments importants de la dynamique de succès.

Nous relèverons tout d'abord combien la satisfaction des voyageurs est au centre de la réforme ferroviaire suisse. Les $\mathrm{CFF}$ font de cette satisfaction le tout premier critère de réussite 
de leur entreprise (CFF, 2012b) et témoignent d'une grande transparence sur ce sujet. La mesure de satisfaction de la clientèle donne lieu à un suivi régulier conduit à partir d'une méthodologie multicritère sur un large échantillon d'usagers (CFF, 2012b). Sont mesurés l'information des voyageurs (en cas de dérangement), le bien-être, le personnel (amabilité et compétence) et la qualité de la prestation (horaire, ponctualité, rapport qualité prix). La réforme ferroviaire s'est accompagnée d'une légère hausse de la satisfaction globale qui, aujourd'hui, tous critères confondus, est particulièrement élevée, avec environ trois quarts de voyageurs satisfaits (CFF, 2012b). Toutefois, la croissance soutenue du trafic et les pics de saturation qui en résultent, associée aux économies drastiques imposées par la Confédération à l'opérateur ferroviaire historique, transforment en défi le maintien de la satisfaction à son niveau actuel.

Un autre indice pertinent de cette satisfaction de la clientèle est sa fidélité. Suite à de fortes pressions du Conseil fédéral visant à promouvoir 1'usage du train, les CFF se sont résolus à une politique tarifaire avantageuse pour les abonnés du rail. Ainsi, dès 1987, le prix de l'abonnement demi-tarif a été fortement réduit (de $360 \mathrm{CHF}$ à $100 \mathrm{CHF}$ ). Il en résulta une spectaculaire augmentation du nombre d'abonnés, 660000 en 1984, plus de 2 millions en 1987 et 2345000 en 2011 (CFF, 2012a). Jusqu'à présent inscrit dans une politique volontariste, l'abonnement général a suivi une progression plus spectaculaire encore : marginal dans les années 1990, avec 25000 abonnés, il concerne en 2011 plus de 430000 abonnés. Actuellement, 2,8 millions de Suisses disposent d'un abonnement aux CFF (sur 7,95 millions d'habitants).

Il importe de noter que cette politique tarifaire avantageuse semble toucher à sa fin. Un débat a dernièrement émergé pour permettre une hausse plus soutenue des tarifs ferroviaires, encore soumis en Suisse à l'autorité du Surveillant des prix. En 2012, aucune majorité n'a émergé au Parlement sur cette proposition, mais il pourrait en être autrement dans l'avenir. En outre, alors que les prix du transport ferroviaire étaient jusqu'alors maîtrisés, évoluant moins vite par exemple que dans des pays ayant connu la libéralisation du transport de voyageurs, comme l'Allemagne ou les Pays-Bas, l'année 2010 a été en Suisse marquée par une forte hausse des tarifs et des abonnements voyageurs (Finger et Holterman, 2013).

Difficile enfin, de ne pas évoquer l'importance de l'attachement exprimé par les citoyens helvétiques envers leur système ferroviaire (referendum) et la politique conduite en son nom dans ce secteur. Chacune des grandes étapes de la politique suisse des transports donne lieu à une votation populaire. Ce fut le cas par exemple pour l'initiative des Alpes, Rail 2000, le fonds $\mathrm{FTP}^{12}$ et pour les grands projets ferroviaires (GPF).

$\mathrm{Au}$ total, la réussite de la réforme du transport ferroviaire régional suisse est donc explicable. Elle tient aux dynamiques particulières impulsées par les pouvoirs publics à l'encontre des trois acteurs du système ferroviaire régional. De ce jeu d'acteurs « gagnantgagnant », il nous reste maintenant à dégager quelques enseignements à même d'éclairer la prochaine réforme de la politique ferroviaire régionale en France.

\footnotetext{
12 En Suisse, le fonds de financement des projets d'infrastructure des transports publics est dit « Fonds FTP ». Alimenté par des ressources dédiées et pérennes, il constitue une originalité et un levier particulièrement favorable au financement de projets d'infrastructure ambitieux.
} 


\section{Les implications pour la prochaine réforme de la politique ferroviaire régionale en France}

Nous conclurons cet article par quelques leçons tirées de l'analyse de la régionalisation ferroviaire en Suisse, pour formuler ensuite des propositions à même d'éclairer les prochaines réformes du transport ferroviaire régional en France.

\subsection{Trois enseignements issus des réformes ferroviaires suisses}

L'analyse des réformes ferroviaires entreprises en Suisse nous conduit à énoncer trois enseignements qui caractérisent la nouvelle gouvernance publique de ce secteur.

Premier enseignement : l'excellence ferroviaire a un coût élevé pour la Collectivité : un coût financier, un coût politique et, enfin, un coût managérial.

En tout premier lieu, l'excellence ferroviaire implique un effort financier considérable des contribuables (Masse, 1991), sans équivalent ailleurs (Prud'homme, 2009). Une production ferroviaire de qualité suppose des investissements considérables et continus (ECMT, 1999). La Suisse a su développer des mécanismes de financement astucieux et pérennes, basés sur des ressources dédiées (NERA, 2004). Les contributions publiques en faveur de l'investissement ferroviaire sont une priorité et sont largement supportées par le budget de la Confédération. Mais le contexte actuel d'argent public rare et de croissance économique ralentie font de la question du financement un véritable défi, en Suisse, comme ailleurs (Leuenberger, 2010).

Un coût politique ensuite. La recherche de l'excellence ferroviaire suppose une implication soutenue et constante des pouvoirs publics en tant que « grand assembleur » du système de transport public. En Suisse, la puissance publique (OFT) définit et conduit une politique de transport originale. Basée sur une coordination étroite de tous les modes et sur une programmation, sur longue période, des objectifs et des actions attendues de chaque acteur, elle vise une plus grande durabilité environnementale. L'organisation du système politique suisse, reposant sur un fédéralisme décentralisé et une forte participation démocratique (via le referendum), contribue certainement pour beaucoup à légitimer l'action publique et à crédibiliser les réformes entreprises. La réforme ferroviaire en Suisse a profondément redessiné le schéma institutionnel entre les acteurs, conférant à l'OFT, mais aussi aux offices cantonaux des transports, un rôle clé potentiel de «network manager» (Genoud, 2000).

Un coût managérial enfin. En tant qu'actionnaire unique de la compagnie ferroviaire historique, les pouvoirs publics assurent, en Suisse, pleinement leur rôle de propriétaire. Ils assignent aux CFF des objectifs stratégiques clairs et partagés, contractualisés par des conventions de prestations évaluées régulièrement et, en assument le prix.

Second enseignement. En Suisse, les pouvoirs publics ont manifestement fait le choix d'améliorer l'efficacité et l'efficience du système ferroviaire en prescrivant une forme originale de gouvernance, optant pour la coopération entre acteurs, plutôt que de le soumettre aux forces de la concurrence (Finger et Genoud, 2004; Meier et Meyer, 2011 ; Finger et Holterman, 2013). Les réformes ferroviaires suisses ont conduit à imposer une « contrainte de performance » à l'opérateur ferroviaire historique et, plus globalement à l'ensemble des entreprises de transport public, en généralisant une contractualisation sous la forme de convention de prestations. 
Le trafic régional de voyageurs, concédé aux CFF par les cantons, constitue un cas exemplaire de ce type de gouvernance : absence totale de concurrence sur et pour le marché et, pourtant, d'importants gains d'efficacité et d'efficience. La clé de ces résultats réside dans deux dispositions : l'introduction de contrats incitatifs, de type « net cost », qui font porter le risque industriel aux opérateurs, et la ferme volonté des pouvoirs publics de plafonner les subventions publiques d'exploitation. La concurrence pour le marché est légalement possible, par mise aux concours de l'attribution des concessions, mais cette modalité ne rencontre aucun écho, dans le domaine ferroviaire.

La concurrence n'est pas totalement absente de la gouvernance suisse du transport ferroviaire régional de voyageurs. Une autre forme de concurrence nous semble jouer un rôle considérable dans la performance du système de transport ferroviaire, la concurrence par comparaison (Shleifer, 1985; Auriol, 2000 ; Bouf et Lévêque, 2006). En effet, différence importante avec la France, le chemin de fer suisse est «pluriel », avec la coexistence au côté d'une entreprise nationale, les CFF, de nombreux autres chemins de fer. Cette pluralité permet aux autorités (OFT) de faire du benchmark et d'entretenir une saine émulation entre les réseaux.

Troisième enseignement : une gouvernance globale triangulaire associant étroitement pouvoirs publics, firmes et clientèle. La qualité de la gouvernance publique, bien qu'essentielle, ne saurait suffire à elle-seule. Elle doit, pour réussir, être accompagnée d'une certaine plasticité managériale de l'opérateur historique, mais aussi d'une adhésion renouvelée de la clientèle.

\subsection{Trois défis à relever par les acteurs du transport ferroviaire régional en France}

Pour faire face aux défis de l'avenir du transport ferroviaire régional en France, nous formulerons trois propositions sur la base de notre analyse de la réforme suisse.

1) La première proposition concerne la $\mathrm{SNCF}$ et les régions en tant qu'autorités organisatrices de transport. L'équilibre à trouver ici vise un troc entre une ouverture à la concurrence limitée et graduée, en contrepartie d'un effort de la SNCF en termes de productivité et de transparence. Cette proposition implique de faire évoluer le cadre réglementaire afin de mettre un terme au monopole légal de la SNCF sur le trafic régional (et de rendre le droit français conforme avec le règlement OSP). Elle implique aussi de changer la nature des contrats proposés pour aller vers des formules « net cost», plus responsabilisantes, attribuées dans le cadre d'une délégation de service public. La Suisse atteste que la concurrence sur ou pour le marché n'est pas une condition indispensable à l'obtention de gains d'efficacité ou d'efficience, à partir du moment où l'opérateur ferroviaire historique est disposé à s'inscrire dans une mutation stratégique positionnant la recherche d'efficience productive au cœur de ses pratiques opérationnelles et le client au centre de son activité.

2) La seconde proposition affecte les relations entre l'État et la SNCF/RFF. Il n'y aura pas d'avenir du rail sans une intensification des investissements (de modernisation et de renouvellement) dans le réseau ferré national (ARF, 2011) et sans une réforme profonde de sa gouvernance à l'initiative et sous l'impulsion de l'État (Favin Levêque, 2009). L'arrangement est ici de faire accepter à la SNCF la mise en place d'une régulation forte, par contractualisation d'objectifs, de gains de productivité et de service, évalués régulièrement, en échange de l'engagement de l'État d'investir durablement dans le réseau (Ministère des Transports, 2005) et de faire de la SNCF la holding en responsabilité de 
l'ensemble de la gestion de l'infrastructure. Le choix fait par la Suisse de donner la priorité aux investissements dans des infrastructures ferroviaires de qualité (aux dépens de la route) suppose une adhésion citoyenne forte, au regard de l'effort fiscal consenti, et le choix d'un mode de financement pérenne par la mise en place de ressources dynamiques dédiées. Quant à la gouvernance par la puissance publique des acteurs ferroviaires, la Suisse illustre combien l'État doit tenir ici toute sa place en particulier d'actionnaire par la mise en place d'un pilotage stratégique exigeant. En matière de gestion de l'infrastructure, la Suisse fait le choix d'un système d'intégration verticale du secteur, faisant de l'unicité une condition de sa performance technique.

3) La troisième proposition implique simultanément les voyageurs, la SNCF et les autorités organisatrices, les régions en premier lieu. L'accord consiste à obtenir de la SNCF une amélioration continue de la qualité de service (ponctualité, information) en échange d'une contribution financière accrue des usagers des transports ferroviaires, elle-même validée par les régions. L'exemple suisse montre combien la satisfaction de la clientèle est un défi permanent, qui doit constituer la valeur fondamentale d'une entreprise de transport ferroviaire en charge de missions de service public. Le cas suisse révèle également une tension non résolue par les pouvoirs publics qui, face à l'accroissement du coût pour le contribuable du financement du ferroviaire (Prud'homme, 2009; Dehornoy, 2012), commencent à envisager des hausses de tarifs. Le cadre du TER s'y prête a priori, vu la très faible participation (moyenne) des usagers au coût d'exploitation (et au coût total) du TER ${ }^{13}$.

La SNCF peut-elle accepter ces évolutions? Les Gouvernements en France veulent-ils et peuvent-ils vraiment assumer ces engagements dans la durée? Quelle sera l'attitude des régions en qualité d'autorités responsables des transports publics dans un contexte libéralisé? Enfin, les clients et les contribuables valideront-ils ces augmentations de leurs contributions?

\section{Conclusion}

Le modèle de régionalisation ferroviaire retenu en France (Chauvineau, 2001) n'est aujourd'hui plus soutenable, tant d'un point de vue financier et organisationnel que réglementaire. L'ouverture prochaine à la concurrence du trafic régional va profondément modifier la donne et élargir l'espace des choix possibles.

Face à ces défis, nous avons ici formulé quelques propositions inspirées de l'exemple de la régionalisation ferroviaire suisse. Bien entendu, la France n'est pas la Suisse. Les CFF présentent de nombreuses différences avec la SNCF et le cadre réglementaire suisse n'est que partiellement conforme aux exigences du droit communautaire (Maier-Gyomlay, 2013). En outre, en matière ferroviaire, les évolutions possibles sont lentes, toujours graduelles et fortement dépendantes de logiques de sentier. Ce qui n'exclue pas quelques inflexions. En la matière, la stratégie suisse a été, nous semble t-il, remarquable, soulignant l'importance de la régulation (Nash, 2011) et de la gouvernance publique (Finger et Holterman, 2013).

\footnotetext{
${ }^{13}$ La Cour des Comptes (2009) soulignait combien les régions avaient été enclines à limiter la contribution des usagers du TER pour en favoriser l'attractivité (sociale), dans un contexte de nette amélioration de l'offre. Ainsi, le prix payé par les usagers représentait (en 2005) environ $17 \%$ du coût complet des TER. En moyenne, les voyageurs ne supportent directement qu'un tiers du coût d'exploitation des TER.
} 


\section{Bibliographie}

AURIOL E., (2000). La concurrence par comparaison : un point de vue normatif. Revue Économique 51, 621634.

BLUMENTHAL P., (1998). Trafic voyageurs régional (TRV) : évolution et perspectives aux CFF. Rail international 4, 2-7.

BOUF D., LÉVÊQUE J., (2006). Concurrence par comparaison pour les services d'infrastructure de transport In ECMT, L'offre de transports : les limites de la (dé)réglementation. Table ronde 129, ECMT, 2006, Paris.

BOVY P.H., (1992). Le modèle ferroviaire suisse : un modèle à suivre? Les Cahiers scientifiques du transport $25,47-66$

BOVY P.H., (1994). L'expérience suisse de l'intermodalité. Transports 365, 187-194.

CARRON N., (2004). La politique ferroviaire dans le cadre de la politique générale des transports en Suisse. Rail International 35, 17-29.

CER, (2005). Reforming Europe's Railways - An Assessment of Progress. Brussels.

CER, (2011). New Reforming Railways - Learning from Experience. Brussels.

CROZET Y., DESMARIS C., (2011). Le transport ferroviaire régional de voyageurs : un processus collectif d'apprentissage. Recherche Transports Sécurité 27 (3), 143-162.

DEHORNOY J., (2012). Les contributions publiques au secteur ferroviaire dans cinq pays d'Europe. Revue Générale des Chemins de Fer 217, 6-18.

DURANTON S. et al., (2012). The 2012 European Railway Performance Index : Understanding what Drives High Performance by European Railways. Boston Consulting Group.

ECMT, (1998). Rail Restructuring in Europe. OECD, Paris.

ECMT, (1999). Investment in Transport Infrastructure 1985-1995. OECD, Paris.

FAVIN LÉVÊQUE J.-C., (2009). Concurrence ferroviaire. La France peut-elle gagner? Lignes de repères, Paris.

FINGER M., GENOUD C., (2004). Concurrence dans les chemins de fer : une chimère? EPFL, MIR, Lausanne.

FINGER M., HOLTERMAN M., (2013). Incentive-based Governance of the Swiss Railway Sector, Florence School of Regulation.

GENOUD C., (2000). La régionalisation des transports publics : implications de la nouvelle loi sur les chemins de fer à l'exemple des cantons de Berne, Zurich, Neuchâtel et Jura. Lausanne, Cahier 188, IDHEAP.

HAENEL H., (2011). Régionalisation ferroviaire : les clés d'un succès. Ville Rail et Transports.

KESELJEVIC C., MEILLASSON S., (2001). Le ferroviaire en Suisse. Rail 84,14-22.

LEUENBERGER M., (2010). L'âme ferroviaire de la Suisse, Conférence des cadres des CFF du 24 juin, Bâle, DETEC.

MASSE J.-P., (1991). Spécial Suisse. Le paradis a un prix. Vie du Rail 2325, 4-20.

MAIER-GYOMLAY J., (2013). Learning Lessons from Switzerland - A BLS perspective. Railway Gazette International January, 36-40.

MEIER B., MEYER A., (2011). Switzerland, In CER, New Reforming Railways - Learning from Experience, Brussels, 127-135.

NAHRATH S., RIEDER M. et al.,(2008). Les impacts de la régionalisation et de la libéralisation sur la durabilité du secteur ferroviaire en Suisse. Flux 72/73, 49-64.

NASH C., (2011). Developments in European Railway Policy. Network Industries Quarterly 13 (1), 11-13.

NERA ,(2004). Study of the Financing and Public Budget Contributions to Railways, Final report for the European Commission, January, London.

OECD, (2006). Regulatory Reform in Switzerland - Regulatory Authorities for Air Transport, Railways, Telecommunications and Postal Services. Paris. 
PFLIEGER G., (2008). Le maillage suisse - La Suisse est-elle un espace première classe ? Flux 72/73, 4-12.

PRUD'HOMME R., (2009). Du modèle ferroviaire suisse. Transports 457, 304-306.

SHLEIFER A., (1985). À Theory of Yardstick Competition. Rand Journal of Economics 20, 41-58.

TORREGO C., GRAFFAGNINO T., (2012). Le système cadencé suisse : caractéristiques essentielles et impacts en matière de production. Revue Générale des Chemins de Fer 219, 36-50.

WEIBEL B., (2012). Le projet Rail 2000. Revue Générale des Chemins de Fer. 219, 52-54.

\section{Annexe des sources documentaires et statistiques}

\section{Sources documentaires (rapports, textes de loi)}

ABRAHAM C., (2011). L'ouverture à la concurrence du transport ferroviaire régional. Rapports et documents, CAS, La Documentation française, Paris.

Accord entre la Confédération suisse et la Communauté européenne sur le transport de marchandises et de voyageurs par rail et par route, dit ATT (Accord sur les transports terrestres) (1999), RS 0.740.72.

ARF, (2011). Manifeste des régions sur le transport de voyageurs. ARF, Paris.

ASSISES DU FERROVIAIRE : assemblée plénière du 15 décembre 2011, clôture des travaux des commissions, Rapport des 4 commissions, site du MEDDTL.

CE, Directive 91/440 du Conseil du 29 juillet 1991 relative au développement des chemins de fer communautaires, JOUE L 23 du 24 août 1991.

CE, Règlement 1370/2007 du Parlement européen et du Conseil du 23 octobre 2007 relatif aux services publics de transport de voyageurs par chemin de fer et par route (dit règlement OSP), JOUE L 315/1 du 3 décembre 2007.

CE, COM2013/25. Le quatrième paquet ferroviaire. L'achèvement de l'espace ferroviaire unique européen pour favoriser la compétitivité européenne et la croissance, Bruxelles.

CESE (2012). L'ouverture à la concurrence des services régionaux de voyageurs, Avis de MM. Jean-Marie GEVEAUX et Thierry LEPAON, 22 juin, JORF.

CFF (2004). Les CFF à plein régime, $\mathrm{CFF}$, Berne.

CFF (2005). Rapport de gestion 2004, CFF, Berne.

CFF (2009). Rapport de gestion 2008, CFF, Berne.

CFF (2012b). CFF : Rapport de gestion et de développement durable 2011. CFF, Berne.

CHAUVINEAU J., (2001). La régionalisation ferroviaire, avis et rapport du Conseil économique et social, séance du 26/09/2001, Paris.

COUR DES COMPTES (2009). Le transfert aux régions du transport express régional (TER) : un bilan mitigé et des évolutions à poursuivre. Rapport public thématique, Documentation française, Paris.

GRIGNON F. (2011). Rapport sur les Conditions pour une expérimentation portant sur l'ouverture à la concurrence des services de transports ferroviaires régionaux de voyageurs, Documentation française, Paris.

HAENEL H. (2008). Écrire l'acte II de la révolution ferroviaire régionale. Rapport au Premier ministre, 26 octobre. Documentation française, Paris.

LCdF. Loi fédérale du 20 décembre 1957 sur les chemins de fer. RS.742.101.

LCdF. Loi fédérale sur les chemins de fer, modification du 24 mars 1995. RS.742.101.

LCdF. Loi fédérale sur les Chemins de fer du 20 mars 1998. RS.742.31.

LITRA, (2008). Le point sur la politique suisse des transports. Berne. 
LITRA, (2011c). L'efficience dans les transports publics - Exemples pratiques. Série jaune. Berne.

LTV. Loi fédérale du 20 mars 2009 sur le transport de voyageurs. RS.754.1.

MINISTÈRE DES TRANSPORTS, (2005), Audit sur l'état du réseau ferré national français, ss. la dir. et la coordination des professeurs Robert Rivier et de Yves Putallaz, Ecole Polytechnique Fédérale de Lausanne.

OFT, (1998). La réforme de chemins de fer : les transports publics sur de nouvelles voies, Berne.

OIPAF. Ordonnance du 18 décembre 1995 sur les indemnités, les prêts et les aides financières selon la loi sur les chemins de fer. RS 742.101.1.

OITRV. Ordonnance du 11 novembre 2009 sur l'indemnisation du trafic régional de voyageurs. RS. 745.16.

SETRA (2009). Le transport ferroviaire de voyageurs sur le réseau ferré national français - Exploration d'un système complexe, Synthèse des connaissances, Ministère de l'Ecologie, de l'Energie, du Développement Durable et de la Mer, septembre.

SRU. Loi 2000-1208 du 13 décembre 2000 relative à la solidarité et au renouvellement urbains, JORF du 14 décembre 2000.

TRV. Directive du 10 décembre 2010 sur la rentabilité minimale dans le trafic régional de voyageurs.

\section{Sources statistiques}

CFF (2012a). CFF : Faits et chiffres 2011. CFF, Berne.

CGDD (2011), Les comptes des transports en 2010, SoeS, Paris.

LITRA (2011a). Les transports en chiffres, Edition 2011. Berne.

LITRA (2011b), Financement de l'exploitation et entretien du réseau ferroviaire, DOKU, 21 mars, Berne.

LITRA (2011d), La statistique ferroviaire 2010 de l'UIC, DOKU, 3 août, Berne.

OFS (2005). Le Compte ferroviaire suisse 2003, OFS, Neuchâtel.

OFS (2009). Le Compte des transports - Année 2005, OFS, Neuchâtel.

OFS (2010). Le Compte ferroviaire suisse 2008, OFS, Neuchâtel.

OFS (2011). Transports publics - Séries chronologiques 1990,1995, 2000-2010. Neuchâtel.

OFS (2012). La mobilité en Suisse - Résultats du micro-recensement mobilité et transports 2010, Neuchâtel.

UIC (2007). Statistiques chronologiques des Chemins de fer 1970-2005, Paris.

UIC (2011). Statistique Internationale des Chemins de Fer 2010, Paris.

UTP (2008), Les transports publics en Suisse, Berne. 